\title{
SOFTWARE LIVRE COMO APOIO À TOMADA DE DECISÃO NO ENSINO E USO DE TECNOLOGIAS: ESTUDO DE CASO PARA AS ESCOLAS PÚBLICAS MUNICIPAIS DE VIDEIRA-SC
}

http://dx.doi.org/10.5902/2318133821338

\author{
Leila Lisiane Rossi \\ Gilson Ribeiro Nachtigall \\ Angela Maria Crotti da Rosa \\ Tiago Heineck \\ Caroline Vian \\ Jeferson José Schneider Boesing \\ Instituto Federal Catarinense - campus de Videira, Brasil. \\ Luiz Gustavo Moro Senko \\ Instituto Federal Catarinense - campus de Brusque, Brasil.
}

\begin{abstract}
Resumo
Neste artigo apresenta-se a ferramenta web desenvolvida para auxiliar na análise dos dados relacionados a uma pesquisa realizada sobre o uso e necessidade de tecnologias no ensino nas escolas públicas municipais urbanas de Videira-SC. Inicialmente foram aplicados questionários dirigidos aos alunos e aos professores e, posteriormente, foi desenvolvida a ferramenta usando software livre com o objetivo de permitir a visualização dos dados de forma multidimensional, ou seja, várias dimensões e diferentes níveis de detalhamento para facilitar a análise dos dados na tomada de decisão.

Palavras-chave: software livre, tomada de decisão, tecnologia.
\end{abstract}

\section{FREE SOFTWARE AS SUPPORT IN DECISION MAKING WHEN USING AND TEACHING TECHNOLOGIES: CASE STUDY IN THE PUBLIC SCHOOLS OF VIDEIRA CITY}

\begin{abstract}
This paper describes the web tool to support the data analysis in the research project about the technological needs in the public schools from Videira city. At first, questionnaires were applied to teachers and students and then, the tool was created using free software allows the multidimensional data visualization, which means, in other words, different dimensions and levels of details which makes it easier to analyze data in the decision making process.

Key-words: free software, decision making, technology.
\end{abstract}




\section{Introdução}

uso de tecnologias está presente em nossas vidas, seja no trabalho, na escola ou em casa, o que nos torna cada vez mais dependentes delas. Em todas as áreas é possível encontrar tecnologia de uma forma ou de outra, o que não é diferente na educação, seja esta na modalidade a distância ou presencial.

Conhecer e saber usar a tecnologia em prol da educação pode contribuir para a melhoria da qualidade, facilidade e rapidez no acesso ao conhecimento e à informação. Com o objetivo de contribuir com as escolas públicas municipais de Videira-SC, foi realizada uma pesquisa com professores e alunos para conhecer as tecnologias usadas nas aulas, bem como as dificuldades no manuseio dos equipamentos e o interesse em cursos gratuitos de informática.

A partir dos resultados obtidos foi criada uma ferramenta web que permite analisar os dados de maneira amigável, facilitando a tomada de decisão em relação à adoção de políticas educacionais. O presente artigo é apresentado conforme segue: na seção 2 apresenta-se uma breve introdução sobre inclusão digital. Na seção 3 são apresentadas as escolas públicas municipais urbanas de Videira-SC. O estudo de caso é apresentado na seção 4. Na seção 5 são descritos os resultados obtidos. A conclusão e os trabalhos futuros são apresentados na seção 6 e, finalmente, as referências usadas aparecem na seção 7.

\section{Inclusão digital}

A inclusão digital é um processo que deve contribuir para a melhoria da qualidade de vida das pessoas, proporcionando facilidades como o aperfeiçoamento profissional e o acesso à informação. As gerações nascidas posteriormente à década de 1780?? são consideradas nativos digitais pela facilidade de acesso e uso das tecnologias. Por outro lado, as demais gerações que, geralmente, possuem algum tipo de dificuldade e que, de uma forma ou de outra, procuram se adaptar a elas e aprendem a usá-las são os chamados migrantes digitais (Prensky, 2001).

Apesar das facilidades dos chamados nativos digitais, sabe-se da situação de grande parte das escolas públicas em relação à dificuldade de acesso às tecnologias e da falta de apoio técnico. Diante deste cenário é importante a realização de parcerias com outras instituições de ensino por meio de projetos de extensão. Acredita-se que o uso adequado de tecnologias nas escolas é relevante, tanto para os professores, ao contribuir para a capacitação profissional, quanto para os alunos, ao proporcionar a oportunidade de aprendizado de forma dinâmica e interativa.

Assim, para a realização da pesquisa foram consideradas todas as escolas públicas municipais urbanas de Videira-SC, no total de seis: Valdemar Kleinubing, Pedro Kleinubing, Caic, Paulo Fioravante Penso, Joaquim Amarante e Antônio Fantin. O foco em escolas públicas, inicialmente as municipais, deve-se ao fato de que, geralmente, estas são mais carentes de recursos tecnológicos.

\section{Informática na escola - estudo de caso}

Nas pesquisas realizadas não foram encontrados trabalhos correlatos, ou seja, nenhuma ferramenta no modelo de um data webhouse - grande armazém de dados na web - para consultas analíticas sobre o uso e a necessidade de tecnologias nas escolas. 
Acredita-se que existam pesquisas relacionadas ou similares, mas possivelmente não encontradas pela forma diferenciada de armazenamento, recuperação e apresentação dos dados. O presente trabalho está sendo desenvolvido no IFC-Videira como um projeto de pesquisa e tem como objetivo conhecer o perfil das escolas públicas municipais de Videira-SC em relação ao uso e a necessidade do conhecimento de tecnologias.

As ferramentas analíticas usadas para o desenvolvimento da ferramenta foram o Pentaho BI Server (Pentaho, 2015), para o Data Webhouse, e a Weka (Weka, 2016) para a mineração de dados. Desenvolveu-se e aplicou-se, inicialmente, um questionário aprovado pelo Comitê de Ética, para a entrada de dados a partir das respostas obtidas nas escolas. Com base neste formulário as informações foram carregadas para a base de dados relacional (Elmasri; Navathe, 2011), pela ferramenta PostgreSQL (Milani, 2008).

$\mathrm{Na}$ sequência o modelo Multidimensional Estrela (Kimball, 2002) foi escolhido para a criação do cubo, permitindo, assim, a disponibilização dos dados em uma tabela Fato centralizada, a qual contém as medidas necessárias para a realização das consultas Analíticas online - Olap - (Inmon, 1996) e várias tabelas Dimensão ligadas a ela. O cubo Professor possui várias tabelas Dimensão, sendo que aqui serão destacadas as principais delas: trabalha com último ano do ensino fundamental, usa computador ou similar nas aulas, dificuldades dos alunos ao usar computador, possui incentivo a capacitação, possui ajuda de profissional de tecnologia, faria curso de tecnologia gratuito, entre outras.

A tabela Fato Professor apresenta as medidas necessárias para a realização das consultas Olap, tais como a quantidade de professores que trabalham com o último ano fundamental, a quantidade de professores que possuem ajuda de profissional de informática, entre outras, ou seja, funções de agregação/medidas relacionadas às tabelas dimensão do cubo Professor. O cubo Aluno possui Dimensões: se o aluno usa computador ou similar nas aulas, se o aluno usa laboratório de informática na escola, se os professores usam tecnologias nas suas aulas, entre outras. A tabela Fato Aluno apresenta as medidas necessárias para a realização das consultas Olap, tais como a quantidade de alunos que usam computador ou similar, a quantidade de alunos que fariam um curso de tecnologia gratuito, outras, ou seja, funções de agregação/medidas relacionadas às tabelas dimensão do cubo Aluno.

A partir do modelo multidimensional armazenado no PostgreSQL (Postgresql, 2016), foi gerado o cubo com a Extensible Markup Language - XML - usando a ferramenta Schema Workbench (Pentaho, 2016). Pelo cubo XML é possível criar consultas Olap e visualizá-las de forma multidimensional na web com a ferramenta Pentaho BI Server (Pentaho, 2016). Estas ferramentas foram escolhidas por serem software livre, seguindo, portanto, as recomendações do governo federal. A consulta Olap é realizada com auxílio da linguagem de manipulação de dados Multidimensional Expressions - MDX. Trata-se de uma linguagem semelhante à Structured Query Language - SQL - (Silberschatz, 2006), porém, utilizada especificamente em Data Webhouse. A ferramenta de mineração de dados Weka (Weka, 2016), aplicada no cubo gerado, permitiu resultados de associação e clusterização considerados importantes para avaliar as preferências e necessidades das escolas.

A figura 1, a seguir, apresenta o uso do laboratório de informática pelos alunos por escola, sendo como resposta "sim" para aqueles que usam e "não" para os que não usam. 
Figura 1 -

Uso de laboratório de informática pelos alunos por escola.

\begin{tabular}{|c|c|c|}
\hline escola & usa_lab_info_escola & - total \\
\hline \multirow[t]{3}{*}{$\exists$ All escolas } & - All usa_lab_info_escolas & 117 \\
\hline & Nao & 78 \\
\hline & Sim & 39 \\
\hline \multirow[t]{3}{*}{ Antonio Fantin } & All usa_lab_info_escolas & 16 \\
\hline & Nao & 2 \\
\hline & Sim & 14 \\
\hline \multirow[t]{2}{*}{ CAIC } & - All usa_lab_info_escolas & 19 \\
\hline & Nao & 19 \\
\hline \multirow[t]{2}{*}{ Joaquim Amarante } & $\begin{array}{r}- \\
\text { All usa_lab_info_escolas }\end{array}$ & 18 \\
\hline & Nao & 18 \\
\hline \multirow[t]{3}{*}{ Paulo Penso } & - All usa_lab_info_escolas & 20 \\
\hline & Nao & 17 \\
\hline & Sim & 3 \\
\hline \multirow[t]{2}{*}{ Pedro Kleinubing } & All usa_lab_info_escolas & 20 \\
\hline & Sim & 20 \\
\hline \multirow[t]{3}{*}{ Waldemar Kleinubing } & EAll usa_lab_info_escolas & 24 \\
\hline & Nao & 22 \\
\hline & Sim & 2 \\
\hline
\end{tabular}

Por uma consulta Olap, como no caso da figura 1, é possível visualizar os dados por níveis de detalhamento - Drill-Down-Up -, ou seja, o usuário pode escolher, por exemplo, a resposta por escola separadamente ou o total geral.

\section{Resultados obtidos}

Como resultado criou-se a ferramenta web pela qual é possível conhecer e analisar facilmente o uso de tecnologias e as preferências dos alunos e professores das escolas pesquisadas. A figura 2 mostra que a maioria dos professores entrevistados não possuem ajuda de profissional de informática nas atividades desenvolvidas no laboratório. Provavelmente pelo fato da maioria das escolas não possuírem profissionais dedicados exclusivamente à área de tecnologia da informação. 
Figura 2 -

Consulta Olap - ajuda profissional de informática.

\begin{tabular}{|c|c|c|}
\hline & & Measures \\
\hline tem_ajuda_profissional_informatica & trabalho_ultimo_ano_fundamental & - total \\
\hline - All tem_ajuda_profissional_informaticas & +All trabalho_ultimo_ano_fundamentals & 23 \\
\hline Nao & + All trabalho_ultimo_ano_fundamentals & 21 \\
\hline Sim & + All trabalho_ultimo_ano_fundamentals & 2 \\
\hline
\end{tabular}

A figura 3 apresenta o resultado sobre a preferência por aulas com o uso de tecnologias. Foram aplicadas algumas técnicas de mineração de dados (Witten; Frank, 2005) como as regra de associação - algoritmo Tertius usando a ferramenta Weka (Weka, 2016) e outras continuam sendo testadas. Pelas regras de associação geradas é possível identificar, por exemplo, que os alunos que tem preferência por aulas com tecnologia também tem interesse em fazer um curso de informática gratuito.

Figura 3 -

Consulta Olap - preferência por aulas com tecnologias.

\begin{tabular}{|c|c|c|c|}
\hline & & & Measures \\
\hline prefere_aulas_com_uso_tecnologias & professores_usam_tecnologias_nas_aulas & usa_lab_info_escola & - total \\
\hline EAll prefere_aulas_com_uso_tecnologiass & † All professores_usam_tecnologias_nas_aulass & ${ }_{\text {All usa_lab_info_escolas }}$ & 117 \\
\hline Nao & ${ }^{\dagger}$ All professores_usam_tecnologias_nas_aulass & ĐAll usa_lab_info_escolas & 11 \\
\hline Sim & ${ }^{\dagger}$ All professores_usam_tecnologias_nas_aulass & † All usa_lab_info_escolas & 106 \\
\hline
\end{tabular}

A figura 4 apresenta a consulta Olap sobre os recursos computacionais nas escolas pesquisadas. É possível verificar que a maioria dos professores consideram que os recursos não são suficientes para as suas aulas. Na figura 5 a consulta Olap apresenta as respostas dos alunos sobre como os professores poderiam usar o computador nas aulas. A maioria das respostas foi a preferência pelo uso do equipamento para realizar pesquisas na Internet.

Figura 4 -

Consulta Olap - recursos computacionais suficientes.

\begin{tabular}{|c|c|c|c|}
\hline & & & Measures \\
\hline recursos_computacionais_suficientes & tem_ajuda_profissional_informatica & trabalho_ultimo_ano_fundamental & - total \\
\hline All recursos_computacionais_suficientess & † All tem_ajuda_profissional_informaticas & ${ }^{ \pm}$All trabalho_ultimo_ano_fundamentals & 23 \\
\hline Nao & †All tem_ajuda_profissional_informaticas & ĐAll trabalho_ultimo_ano_fundamentals & 13 \\
\hline Sim & ${ }_{\text {† All tem_ajuda_profissional_informaticas }}$ & ¿ All trabalho_ultimo_ano_fundamentals & 10 \\
\hline
\end{tabular}


Figura 5 -

Consulta Olap - como o professor poderia usar computador nas aulas.

\begin{tabular}{|l|r|}
\hline Como_professor_poderia_usar_computador_aulas & $\bullet$ total \\
\hline All como_professor_poderia_usar_computador_aulas & 117 \\
\hline Jogos_Educativos & 20 \\
\hline Outras_Formas & 11 \\
\hline Pesquisas_Internet & 50 \\
\hline Videos & 36 \\
\hline
\end{tabular}

A figura 6 apresenta um exemplo de consulta analítica pela qual é possível identificar que a maioria dos professores declaram não terem ajuda de um profissional de tecnologia e que a maioria faria um curso gratuito de informática. Vale ressaltar que os professores não foram questionados sobre o motivo de não possuírem ajuda em relação ao uso de tecnologias, mas possivelmente seja por não terem profissionais exclusivos para o cargo ou tarefa.

Figura 6 -

Consulta Olap - ajuda profissional/curso tecnologia.

\begin{tabular}{|c|c|c|c|}
\hline & & & Measures \\
\hline faria_curso_tecnologia_gratuito & recursos_computacionais_suficientes & tem_ajuda_profissional_informatica & - total \\
\hline - All faria_curso_tecnologia_gratuitos & ${ }^{\dagger}$ All recursos_computacionais_suficientess & (4) All tem_ajuda_profissional_informaticas & 23 \\
\hline Nao & $\biguplus_{\text {All recursos_computacionais_suficientess }}$ & ${ }^{+}$All tem_ajuda_profissional_informaticas & 3 \\
\hline $\mathrm{Sim}$ & ${ }^{\dagger}$ All recursos_computacionais_suficientess & EAll tem_ajuda_profissional_informaticas & 20 \\
\hline & & Nao & 18 \\
\hline & & Sim & 2 \\
\hline
\end{tabular}

Pelas consultas realizadas na ferramenta web pode-se obter as seguintes informações sobre as escolas públicas municipais de Videira-SC: $91 \%$ dos professores declararam que não possuem ajuda de profissional de informática; $91 \%$ dos alunos fariam curso de tecnologia gratuito; $90 \%$ dos estudantes preferem aulas com tecnologias; $57 \%$ dos professores consideram que os recursos computacionais não são suficientes.

A partir de consultas como estas é possível tomar algumas decisões como, por exemplo, a adoção de políticas para ofertar cursos de tecnologias para as escolas em parceria com outras instituições de ensino. Com base nos questionários aplicados os resultados mostram a grande necessidade de profissionais de informática ou de programas de capacitação dos professores, uma vez que a pesquisa revelou que muitos gostariam e precisariam melhorar o conhecimento em relação ao uso de tecnologias. Essas e outras melhorias poderiam ser realizadas por parcerias.

\section{Conclusão e trabalhos futuros}

A ferramenta desenvolvida permite realizar consultas Olap na web sobre os dados dos alunos e professores de escolas públicas municipais de Videira-SC em relação ao uso/necessidade de tecnologias. A ferramenta oferece a possibilidade de analisar os 
dados por uma interface amigável e para facilitar a tomada de decisão por parte dos coordenadores, diretores e envolvidos na gestão em geral. Vale destacar que outras ferramentas de estatística mais simples como, por exemplo, a planilha eletrônica poderia ter sido escolhida para a realização da pesquisa, considerando que o volume de dados obtidos das escolas é relativamente baixo. Porém, com as ferramentas usadas é possível visualizar os dados de várias dimensões, além de facilitar a expansão e a apresentação de novas informações no caso de continuidade da pesquisa.

Como trabalhos futuros pode-se propor uma pesquisa mais ampla sobre as escolas, aumentando a utilidade da ferramenta. Além disso, poderia ser pesquisado sobre as demais escolas públicas do município, possivelmente as estaduais ou mesmo outras instituições que tenham interesse. A aplicação de outras técnicas de mineração dados, possivelmente sobre um questionário ampliado, também poderiam ser incrementadas possibilitando a descoberta de padrões desconhecidos na base de dados. Pelos resultados obtidos poderia ser proposto algum curso ou parceria do Instituto com essas escolas, conforme interesse e disponibilidade de ambas as partes, uma vez que a pesquisa mostra a necessidade e o interesse de capacitação, tanto dos alunos, quanto dos professores.

\section{Referências}

KIMBALL, Ralph. Data warehouse toolkit: o guia completo para modelagem multidimensional. Rio de Janeiro: Campus, 2002.

ELMASRI, Ramez; NAVATHE, Shamkant B. Sistemas de banco de dados. São Paulo, Pearson, Addison Wesley, 2011.

MILANI, André. PostgreSQL: guia do programador. São Paulo, Novatec, 2008.

PENTAHO. Pentaho open source business intelligence. Disponível em $<$ http://www.pentaho.com>. Acesso em 18 fev. 2016.

POSTGRESQL. PostgreSQL. Disponível em <http://www.postgresql.org>. Acesso em 18 fev. 2016.

PRENSKY, Marc. Digital natives, digital immigrants - 2001. Disponível em shttp://www.marcprensky.com/writing/Prensky-Digital Natives,Digital ImmigrantsPart1.pdf >. Acesso em 18 fev. 2016

SILBERSCHATZ, Abraham; KORTH, Henry F; SUDARCHAN, S. Sistemas de banco de dados. São Paulo: Makron Books, 2006.

WEKA, Pentaho. Open source business intelligence - data mining. Disponível em <http://community.pentaho.com/projects/data-mining/>. Acesso em 18 fev. 2016.

WITTEN, Ian H; FRANK, Eibe. Data mining: practical machine learning tools and techniques. San Francisco: Elsevier, 2005.

Leila Lisiane Rossi é mestre em Ciência da Computação e professora de Informática no Instituto Federal Catarinense - campus de Videira.

Endereço: Instituto Federal Catarinense - campus de Videira - rodovia SC 135, Km 125 - 89560-000 - Videira - SC - Brasil.

E-mail: leila.rossi@ifc-videira.edu.br. 
Gilson Ribeiro Nachtigall é doutor em Agronomia, coordenador de pesquisa e inovação e professor dos cursos de Agropecuária no Instituto Federal Catarinense - campus de Videira.

Endereço: Instituto Federal Catarinense - campus de Videira - rodovia SC 135, Km 125 - 89560-000 - Videira - SC - Brasil.

E-mail: gilsonrn@ifc-videira.edu.br.

Luiz Gustavo Moro Senko é mestre em Ciência da Computação e professor de Informática no Instituto Federal Catarinense - campus de Brusque.

Endereço: Instituto Federal Catarinense - campus de Brusque - Rua Hercílio Luz, 373 - 88350-301 - Brusque - SC - Brasil.

E-mail: gustavo.senko@brusque.ifc.edu.br.

Angela Maria Crotti da Rosa é especialista em Controle da Gestão Pública e coordenadora de estágios e extensão no Instituto Federal Catarinense - campus de Videira.

Endereço: Instituto Federal Catarinense - campus de Videira - rodovia SC 135, Km 125 - 89560-000 - Videira - SC - Brasil.

E-mail: angela rosa@ifc-videira.edu.br.

Tiago Heineck é especialista em Engenharia de Sistemas e Técnico em Tecnologia da Informação no Instituto Federal Catarinense - campus de Videira.

Endereço: Instituto Federal Catarinense - campus de Videira - rodovia SC 135, Km 125 - 89560-000 - Videira - SC - Brasil.

E-mail: tiago.heineck@ifc-videira.edu.br.

Caroline Vian é tecnóloga em Gestão de Recursos Humanos e coordenadora de Gestão de Pessoas no Instituto Federal Catarinense - campus de Videira.

Endereço: Instituto Federal Catarinense - campus de Videira - rodovia SC 135, Km 125 - 89560-000 - Videira - SC - Brasil.

E-mail: caroline.vian@ifc-videira.edu.br.

Jeferson José Schneider Boesing é técnico em informática e aluno do curso de Ciência da Computação no Instituto Federal Catarinense - campus de Videira.

Endereço: Instituto Federal Catarinense - campus de Videira - rodovia SC 135, Km 125 - 89560-000 - Videira - SC - Brasil.

E-mail: jefersonboesing@yahoo.com.br.

Recebido em 3 de abril de 2016.

Aceito em 19 de junho de 2016. 